\title{
REFLEXIONS SUR LA RESPONSABILITE INDIVIDUELLE ET COLLECTIVE DES CRIMES NAZIS
}

REFLECTIONS ON INDIVIDUAL AND COLLECTIVE RESPONSIBILITY OF NAZIS CRIMES

\section{Rainer Maria Kiesow}

Juriste allemand, professeur de droit privé et directeur d'études à l'École des hautes études en sciences sociales (EHESS). Il est éditeur de la revue Grief. Revue sur les mondes du droit. E-mail: rainermariakiesow@gmail.com

Convidado

RÉSUMÉ: L'objectif de ce bref travail est de reflexions sur la responsabilite individuelle et collective des crimes nazis.

Mots-clés: Responsabilite Individuelle Et Collective. Crimes Nazis. Flussi Migratori. Violazione dei Diritti Umani.

\section{INTRODUCTION}

Il s'est excusé. Il était désolé. Mais il n'a pas avoué. Un monsieur sans aucune trace de monstre dans son visage et ses yeux de 95 ans, qui se sont éteints le 30 mai 2017. Un vieil homme comme les autres. Quand il avait 22 ans, son visage et ses yeux ne laissaient pas non plus voir la complicité de meurtre de 170 000 personnes. Un jeune homme comme les autres. C'était à Auschwitz en 1943 et 1944, et il était gardien SS dans le camp d'extermination. Le 17 juin 2016, Reinhold Hanning a été condamné par le tribunal de grande instance de Detmold à une peine de prison de cinq ans. Le jugement n'était pas définitif, le prévenu est mort avant que la cour de cassation allemande n'ait pu se prononcer sur le recours de l'accusé.

Oskar Gröning a fêté son 96e anniversaire, le 10 juin 2017. C'était l'un des comptables d'Auschwitz; il comptait l'argent des nouveaux arrivés à la rampe et le triait par devises. Il n'a jamais tué personne. Il a été condamné par le tribunal de grande instance de Lüneburg à une peine de prison de quatre ans pour complicité de meurtre de 300000 personnes. Une preuve de plus, s'il en était besoin, que la justice dépend du cas et du juge, et non mécaniquement de la loi. Quatre ans pour 300 000, cinq ans pour 170000 ? Le 20 septembre 2016, la cour de cassation allemande à Karlsruhe maintient le verdict.

On cherche toujours une prison gériatrique où mettre le condamné, étant donné qu'en Basse-Saxe, avant le cas Gröning, le prisonnier le plus âgé n'avait que 83 ans. 


\section{REFLEXIONS SUR LA RESPONSABILITE INDIVIDUELLE ET COLLECTIVE DES CRIMES NAZIS}

Il existe encore quelques rares autres cas relevant de cet ensemble complexe des auxiliaires des crimes nazis, à savoir toutes ces personnes qui n'ont pas été auteurs-tueurs sur le front de la boucherie nazie, mais qui ont œuvré en arrière-plan pour que les auteurs puissent commettre leurs crimes sans problème. Des enquêtes judiciaires ont ainsi eu lieu à Hanau (devant un tribunal de mineurs, puisque le prévenu avait moins de 21 ans à l'époque des faits), à Kiel, à Neubrandenburg. La mort ou la santé des prévenus ont finalement empêché les procès à Hanau et à Kiel. À Neubrandenburg, le procès est en cours.

Depuis 2011, une cinquantaine de procès ont été engagés en Allemagne contre des nonagénaires sur le fondement de la complicité de meurtre. Le signal de départ fut donné le 12 mai 2011 par le jugement du tribunal de grande instance de Munich, quand le gardien John Demjanjuk fut condamné à une peine de prison de cinq ans pour complicité de 28060 meurtres dans le camp d'extermination de Sobibor. C'était la première fois qu'un prévenu, qui, du reste, n'était pas allemand mais d'origine ukrainienne et avait obtenu en 1958 la nationalité américaine, était condamné pour complicité sans que l'on pût prouver de sa part une quelconque action directe et concrète en lien avec le meurtre. Demjanjuk étant décédé avant que la cour de cassation allemande ne se soit prononcée, le verdict n'a pu être confirmé ou infirmé. Mais la course était déclenchée. Car, enfin, une cour de justice, celle de Munich, a bouleversé le droit et cela, dans un secteur extrêmement sensible du droit pénal, c'est-à-dire la zone entre l'acte et le non-acte, entre la qualification d'auteur-acteur et de celui qui n'a rien fait, bref, la zone dans laquelle se trouvent ceux qui n'ont pas rien fait, mais qui n'ont pas non plus commis l'acte, donc ceux qui y contribuent. C'est la zone intermédiaire entre l'interdit et le permis, cette zone où le complice fait son œuvre.

Le droit pénal de la complicité est (avec celui de la tentative) certainement l'un des plus complexes en droit. Il s'agit de poser une différence normative et nominaliste entre le rien et le début de quelque chose. Pour ne pas sombrer dans le tout et n'importe quoi, le droit a forgé au cours des siècles des instruments techniques interprétatifs qui ont abouti, dans le droit pénal du XIX ${ }^{\mathrm{e}}$ siècle, en France comme en Allemagne, à des principes généraux comme le caractère nécessairement accessoire (de l'acte du complice par rapport à l'acte principal), la causalité, l'immédiateté, la proximité. Ce sont des garde-fous pour ne pas impliquer tout un chacun dans un acte maléfique. En résumé : la complicité au sens pénal doit être liée à l'acte, le complice doit savoir spécifiquement en quoi consiste l'acte principal, il doit y contribuer concrètement. Un individu qui donne un couteau de cuisine à un autre en sachant vaguement que cet individu a des velléités brutales n'est pas forcément complice si celui-ci tue quelqu'un avec ledit couteau de cuisine. Ce serait peut- 
être différent si l'arme du crime était un poignard. Comme toujours dans le droit : cela dépend - de nombreux éléments du cas. Si les prérequis de causalité et d'immédiateté se sont effrités au cours du temps, en principe il ne suffit pas - il ne suffisait pas -, pour être complice, d'être simplement là où le crime d'un auteur a eu lieu.

Le jugement « Demjanjuk » et, surtout, la décision définitive « Gröning » de la cour de cassation ont changé ces critères doctrinaux et jurisprudentiels. Maintenant, c'est tout différent, radicalement autrement. Comme l'énonce la cour de cassation : « [le prévenu] était intégré dans l'organisation des homicides en masse en assurant des tâches, d'après le tableau de services, à l'arrivée des victimes à la rampe », autrement dit : « il faisait partie de l'appareil du personnel en service à Auschwitz ».

Formulé d'une manière aussi laconique que spectaculaire dans ses conséquences juridiques, cet énoncé signifie que le lien entre la complicité et l'acte peut être établi par une pure fonctionnalité. Plus besoin d'un lien concret, individuel, immédiat, proche, etc. Il suffit de prouver que le complice occupait une fonction dans l'appareil. Et bien sûr, mais cela les prévenus eux-mêmes ne l'ont pas nié, il est clair que cet appareil n'était pas une fabrique de bonbons, mais de morts. À partir de cette jurisprudence nouvelle, à partir de 2011-2016 donc, tous les agents formant le personnel des camps de concentration, fussent-ils gardiens, bureaucrates ou cuisiniers, restés en poste un jour ou un an, sont susceptibles d'être qualifiés et condamnés comme complices d'au moins un meurtre. Pour mémoire : dans le seul camp d'Auschwitz-Birkenau, ce sont environ 10000 hommes et femmes qui ont travaillé comme personnel SS.

Il était temps. Le temps passe. Le droit est une question de temps. C'est cruellement le cas si la responsabilité des crimes nazis est en jeu. Car ne nous leurrons pas : le champ entre le rien et l'acte, id est le champ de la complicité, c'est le champ de la responsabilité de beaucoup de personnes, de toutes peutêtre.

Car il est assez facile de dire (et de ne pas subir la preuve du contraire) : « Je n'ai tué personne»ce qui veut dire que la frontière entre l'acte et le reste, le meurtre et le non-meurtre, ne pose pas vraiment de problème, sauf à concevoir une masse d'auteurs via la non-assistance à personne en danger, fondement juridique assez tiré par les cheveux dans le cas des crimes de masse. Non, le peuple allemand ne fut pas, ni en réalité ni en termes juridiques, un peuple d'auteurs de crimes.

En revanche, il est difficile de dire : «Je n'ai rien entendu, rien vu, rien fait, je ne faisais partie d'aucune organisation de l'État allemand nazi à quelque niveau que ce soit ». Et maintenant, avec les procès de 2011-2016, nous y voilà : l'intégration dans la zone de la complicité de tous ceux qui n'ont, pour ainsi dire, rien fait, de cette grande majorité qui n'a justement tué personne. Et cette zone devient très, très large, depuis que nous savons qu'il n’y a plus grand monde, en réalité quelques douzaines de cas judiciaires au plus, et quelques dizaines de milliers de personnes très âgées qui avaient autour de 20 ans à l'époque de la 
« solution finale »- depuis que le peuple de jadis, le peuple des complices de crimes, est en voie de disparition. Ce peuple-là est (quasi) mort.

Et c'est précisément à ce moment-là que le peuple ressurgit. Il ne s'agit pas du peuple historique, celui-ci est mort, il ne s'agit pas du peuple actuel, il est concerné par d'autres choses, il s'agit d'un peuple idéel, d'un peuple imaginaire, d'un peuple qui peut prendre et porter le fardeau de la responsabilité, justement parce qu'il est mort. C'est ce que signifie le revirement spectaculaire de cette jurisprudence visant la responsabilité des complices. Le champ de la complicité s'ouvre au moment où presque aucun complice n'est plus vivant. Il s'ouvre à la masse des morts, par définition non justiciables, et aux quelques vieillards qui, avec leurs visages semblables à ceux de tous les hommes au seuil de la mort, suscitent plus de peine que le besoin d'en infliger une.

C'est une question de temps. Le droit est une question de temps, fondamentalement. Le droit tout entier, c'est-à-dire toutes nos relations sociales susceptibles d'être jugées, en matière pénale, privée ou administrative, se trouve sous l'épée du temps. Le terme juridique pour ce moissonneur des espoirs et des craintes relatives au droit et à la justice, c'est la prescription. La prescription permet au temps de faire son œuvre : oublier, excuser, laisser passer. Sans prescription, la responsabilité demeure toujours, peut toujours être tirée de la latence à la lumière ; sans prescription, le droit exerce un pouvoir sans limite, c'est-à-dire total, même soixante-dix ans plus tard.

La prescription, la question de l'arrêt de la course du droit, a donné lieu à ce qu'on appelle jusqu'à aujourd'hui l'« heure de gloire du Parlement », du Bundestag allemand. C'était le 10 mars 1965. Le débat portait sur une question, sur la question du temps. D'après le code pénal en vigueur, le meurtre était soumis à une prescription de 20 ans. Près de vingt ans après la fin de la barbarie, il s'agissait de prolonger (ou pas) les délais. Oublier, excuser, laisser passer ? Nullum crimen, nulla poena sine lege ? Principe de légalité ? Interdiction de la rétroactivité ? Six millions de cadavres, juifs, Tziganes, communistes, homosexuels ? Et les auteurs des actes, les Täter? Le plus grand procès de l'histoire de la République fédérale allemande, le (premier) procès d'Auschwitz devant la cour d'assises de Francfort, était en cours depuis quinze mois. Après les procès à Nuremberg, organisés par les alliés vainqueurs contre les hauts dirigeants nazis, après les quelques procès sous l'occupation et après la création de la Rfa, devrait-on en rester là ? La responsabilité approuvée, jugée pour quelques-uns... et tous les autres acteurs-auteurs qui seraient encore libres, engloutis dans la "société économique en pleine croissance », dans le Wirtschaftswunderland ? Pouvait-on, au bout de vingt ans, tirer un trait (tout à fait légalement au vu du principe fondamental de la non-rétroactivité des lois pénales plus sévères) sur la responsabilité pour les actes atroces du III ${ }^{\mathrm{e}}$ Reich qui, lui, avait vocation à durer mille ans ? Et cette responsabilité, au fait, qui concerne-t-elle ? Toi, moi, vous, nous tous ? 
Karl Jaspers, le célèbre philosophe et psychiatre, ami de Max Weber, ami pour la vie de Hannah Arendt, ami d'avant 1933 de Martin Heidegger, Karl Jaspers qui pensait encore en 1933 que le principe du Führer serait bien pour une réforme de l'université et qui s'est trompé cruellement sur le prétendu caractère d'opérette du régime nazi, Karl Jaspers qui, étant marié à une juive, s'est tôt réfugié dans l'émigration intérieure, Karl Jaspers qui est devenu après la guerre l'une des grandes autorités morales de la vie intellectuelle allemande, avait consacré, en 1946, son cours inaugural à l'Université (refondée) de Heidelberg à la question de la responsabilité, "Die Schuldfrage ». Il y distingue les responsabilités " criminelle, politique, morale et métaphysique ». La responsabilité criminelle est affaire des cours de justice, donc se mesure d'après les lois en vigueur. La responsabilité morale, au-delà des temps juridiques, reste, pour toujours, indépendante d'un traitement judiciaire. Et, la responsabilité, de quelque nature qu'elle soit, ne peut, ne doit pas être collective. Juridiquement ou moralement, la responsabilité demeure affaire de l'individu, du seul individu. Le cours de Jaspers fut certainement l'un des points de départ les plus importants du vaste débat, qui perdure jusqu'à nos jours, de la responsabilité collective concernant les crimes commis sous le III ${ }^{\mathrm{e}}$ Reich.

Et cette question de la responsabilité collective, tout à fait inséparable de la question de la prescription, fut également au centre du débat parlementaire du 10 mars 1965. C'est le juriste Adolf Arndt, l'un des grands hommes politiques du parti social-démocrate $(\mathrm{Spd})$, figure de proue quelques années auparavant du programme réformiste, antisocialiste de Godesberg, c'est Adolf Arndt qui exprima devant l'Assemblée toute l'ambivalence - pour ne pas dire le gouffre - de la responsabilité vis-à-vis de ce qui s'est passé pendant douze ans en Allemagne :

On savait l'essentiel. J'ai dû dire à de jeunes gens : "Si votre mère est sur son lit de mort et vous jure devant Dieu (...) qu'elle ne savait pas, alors je vous dis : votre mère n'arrive simplement pas à le dire, parce que c'est trop horrible de l'avoir su ou d'avoir pu le savoir, mais de n'avoir pas voulu le savoir" (...). Je sais que je porte aussi une responsabilité. Car, voyez-vous, je ne suis pas descendu dans la rue et je n'ai pas crié, quand j'ai vu qu'on saisissait les juifs parmi nous et qu'on les déportait par camions entiers. Je ne me suis pas mis l'étoile jaune sur la poitrine et je n'ai pas dit : "Moi aussi !" (...). Je ne peux pas dire que j'en ai fait assez (...). Il s'agit de ne pas tourner le dos à la montagne de responsabilité et de malheur qui se trouve derrière nous. (Ma traduction). ${ }^{1}$

${ }^{1}$ Cf. Verhandlungen des Deutschen Bundestages, 4. Wahlperiode. Stenografische Berichte Band 57 : 170. Sitzung vom 10. März $1965: 8552$ sq. 
La prescription fut étendue, d'abord, par la nouvelle « Loi relative au calcul des délais de prescription en droit pénal » qui fixait le point de départ du délai de la prescription au 31 décembre 1949, l'année de naissance de la Rfa. On se doute bien que cela ne suffisait pas, car on ne gagnait de la sorte que quatre années. La durée de la prescription fut alors étendue à trente ans et puis, finalement, tout simplement supprimée pour tout meurtre, l'imprescriptibilité s'appliquant également à la tentative et la complicité du meurtre. C'est notamment grâce à l'aide du «Service central d'enquête sur les crimes nationauxsocialistes », entré en activité le 1er décembre 1958 à Ludwigsburg, que des procès contre des nazis peuvent avoir lieu jusqu'à nos jours. La justice allemande a condamné, depuis le 8 mai 1945, moins de 7000 personnes. Dans l'ensemble, il n'y eut que peu, très peu, bien trop peu de procès.

Et maintenant voici la nouvelle jurisprudence sur la complicité. Il suffit d'avoir su qu'il y avait des meurtres et d'avoir été un élément fonctionnel dans une organisation visant au meurtre. L'acte individuel, jusqu'alors tout à fait nécessaire pour concevoir juridiquement la complicité, se fond dans le collectif d'une machine à tuer. Peu importe quel petit rouage représentait l'individu dans cette machine, cela pourrait être le Hundeführer, le « maître-chien », l'éplucheur de légumes aux cuisines ou l'attaché culturel du camp - qui ne sont plus parmi nous.

Mais cette nouvelle jurisprudence va plus loin encore. Elle ne le dit pas, elle ne peut pas le dire, car un cas est un cas, et les obiter dicta, les remarques des juges au-delà du cas d'espèce, sont rares. Mais il est clair que cette jurisprudence conduit à inclure, dans le dispositif de la responsabilité, l'homme moyen entre 1933 et 1945, tous ceux qui ont participé, contribué, concouru au fonctionnement du régi-me, les soldats peut-être en première ligne, les agents des chemins de fer, les fonctionnaires de toutes sortes et de tous niveaux, et même, au-delà de ces attaches étatiques et administratives, les bouchers, les boulangers, les électriciens, tous ceux qui ont œuvré au maintien de la force, de l'opérationnalité du Reich et, plus loin encore peut-être, in extremis, les mères et les pères qui donnaient de nouvelles vies à cet organisme meurtrier. En somme, la nouvelle construction de la complicité juridique concerne en principe tout le monde, car tous, presque tous, étaient un petit rouage dans la grande roue de la mort, et tous étaient au courant, pas dans tous les détails, mais souvenez-vous de ce que Adolf Arndt a dit et de ce qui s'est passé devant les yeux de tous à partir de 1933. Et voilà la responsabilité juridique étendue au niveau collectif, tout en partant d'un cas singulier, mais si le singulier est un singulier dans une mer de singuliers qui sont fonctionnellement comme les autres, le terme singulier n'a plus guère de sens.

[http://dipbt.bundestag.de/doc/btp/04/04170.pdf]. 
Il est évident que cette jurisprudence n'aurait pas pu naître quand ces atomes fonctionnels étaient encore en vie. On ne peut pas condamner un peuple entier, qui entretemps s'est employé à construire la nouvelle démocratie, tachée profondément - et inévitablement ? - par cette ancienne population restée en fonction, pour administrer, pour renseigner, pour juger, et pour électrifier, chauffer, cuisiner. À l'époque, on n'avait même pas réussi, loin s'en faut, à juger tous les auteurs et complices directs des actes meurtriers ; il n'est donc pas étonnant que la complicité accessoire et fonctionnelle n'ait alors pas vu le jour. «Vive le peuple ! », autrement dit «Vive la responsabilité collective ! », cette nouvelle jurisprudence s'appuyant sur une conception fonctionnaliste de la complicité en droit pénal n'a été rendue possible qu'au moment où justement le peuple concerné était mort. Finalement, le temps a eu raison de nos tentatives faiblardes pour nous confronter aux crimes inouïs du milieu du $\mathrm{XX}^{\mathrm{e}}$ siècle. Il n'a, certes, pas excusé, mais il a laissé passer, comme toujours. Et un Nelson Mandela qui a employé, entre la condamnation totale et le laisser-passer total, une troisième voie, celle de la réconciliation extra-juridique, en toute connaissance de cause, avec un passé atroce, n'était pas disponible. Mais, au-delà des cas individuels actuels qui n'ont - mais peut-être je me trompe, notamment eu égard aux victimes - finalement pas une grande importance et qui mènent à d'inévitables bizarreries, comme de chercher des mois et des mois, et pour l'instant en vain, une prison adaptée pour héberger un condamné vieillard et moribond, cette toute dernière jurisprudence sur la complicité a elle-même une signification pour nous, nous les habitants d'aujourd'hui.

C'est qu'elle met le doigt sur le problème de la responsabilité, un problème que chaque génération doit porter pour elle-même dans son esprit et dans son cœur. L'adieu prononcé dans les prétoires de Munich et de Karlsruhe à la responsabilité matérielle, concrète, active, en bref, substantielle, et la création de la complicité fonctionnelle assemblent, ramassent les individus dans un collectif. Il n'est peut-être pas insensé d'y penser, que chacun de nous y pense, quand nous sommes assis devant la télévision, l'ordinateur, le smartphone, assis sur le canapé à la maison, à la terrasse d'un café, dans le transat à la plage, consommant les toutes dernières nouvelles en matière de guerre, de famine, d'inondations et de morts dans le monde entier - et dont il est difficile de dissocier complètement notre fonctionnement propre à nous, ici. A tweet is not enough ! Et il me vient en tête cette phrase de l'officier « Dans la colonie pénitentiaire » de Franz Kafka, cette phrase qui est la base de tout jugement sur cette île lointaine : «La responsabilité ne fait jamais de doute ». La complicité non plus. Et ainsi continuerons-nous de vivre sous l'épée de Damoclès de la complicité. C'est inévitable et cela contribue à construire la conscience de chacun de nous. Mais, au sens large de la fonctionnalité de chacun de nous dans l'ordre existant, cette défaillance collective face aux atrocités actuelles ne sera justiciable (et encore) qu'après nous - quand nous ne serons plus de ce monde. Il est fort probable qu'il ne puisse jamais en être autrement. 